\title{
Seleção de fungos patogênicos para controle de larvas e pupas da mosca-das-frutas Ceratitis capitata (Diptera: Tephritidae)
}

\section{Selection of pathogenic fungi for the control of Ceratitis capitata (fruit fly) larvae and pupae}

\author{
Gustavo BISSOLLI; ${ }^{1 ;}$; Antônia do Carmo Barcelos CORREIA ${ }^{3}$; José Carlos BARBOSA ${ }^{4}$ \\ ${ }^{1}$ Parte da dissertação de mestrado do primeiro autor; Conselho Nacional de Desenvolvimento Científico e \\ Tecnológico - CNPq. \\ ${ }^{2}$ Autor para correspondência; Mestre; Coordenadoria de Defesa Agropecuária - Escritório de Defesa \\ Agropecuária de Jaboticabal, Rua Barão do Rio Branco, 372 CEP: 14870-330; gustavo@cda.sp.gov.br. \\ ${ }^{3}$ Prof. Dr. Aposentada pela Universidade Estadual Paulista (UNESP), Faculdade de Ciência Agrárias e \\ Veterinárias (FCAV). \\ ${ }^{4}$ Prof. Doutor; Universidade Estadual Paulista (UNESP), Faculdade de Ciência Agrárias e Veterinárias (FCAV); \\ E-mail: jcbarbosa@fcav.unesp.br.
}

Recebido em: 13-06-2013; Aceito em: 27-05-2014

\section{Resumo}

A mosca-das-frutas, Ceratitis capitata é considerada praga de grande importância econômica no Brasil e no mundo, sendo considerada também espécie quarentenária em diversos países. Normalmente, são utilizados inseticidas químicos para o controle deste inseto, causando impactos na saúde e no meio ambiente, inclusive podendo haver restrições de países importadores devido à presença de resíduos tóxicos nos frutos. Portanto, há necessidade de estudos de outros métodos de controle, para incrementar o Manejo Integrado de Pragas, usando agentes de controle biológico. $\mathrm{O}$ objetivo deste trabalho foi avaliar a patogenicidade dos fungos entomopatogênicos das espécies Metarhizium anisopliae (Metsch.) Sorokin, Beauveria bassiana (Bals.) Vuillemin, Paecilomyces fumosoroseus Bainier, Paecilomyces sp. Bainier, Verticillium lecanii (Zimm.) Viegas, em larvas de $3^{\circ}$ instar e pupas da mosca-das-frutas Ceratitis capitata Wiedemann (Diptera: Tephritidae). Realizou-se inicialmente uma seleção destes isolados em lanvas, através de imersão em suspensão aquosa de conídios. A mortalidade larval causada por 36 isolados dos fungos variou de $2,5 \%$ a $96,2 \%$. Destacaram-se como mais patogênicos, seis isolados de $M$. anisopliae e dois de $B$. bassiana. Numa segunda série de bioensaios, conduzidos com os isolados selecionados, pupas foram tratadas com suspensão aquosa, na concentração de $1,2 \times 10^{8}$ conídios viáveis $/ \mathrm{mL}$ e germinação variando de 85 a $98,2 \%$. A mortalidade pupal causada pelos fungos foi baixa, variando de 1,9 a $4,4 \%$, porém houve diminuição da longevidade dos adultos sobreviventes.

Palavras-chave adicionais: controle microbiano; fungos entomopatogênicos; mosca-do-mediterrâneo.

\begin{abstract}
The fruit fly, Ceratitis capitata, is considered a pest of great economic importance and is also considered a quarantine species in several countries. Usually chemical insecticides are used for the control of this pest. These products may cause impacts on the public health and on the environment and this leads importing countries to restrict the amounts of imported fruits due to the possibility of toxic residues in the fruits. So, studies of other control methods are necessary to increase the integrated management of pests. The objective of this study was to evaluate the pathogenicity of the entomopathogenic fungi of the species Metarhizium anisopliae (Metsch.) Sorokin, Beauveria bassiana (Bals.) Vuillemin, Paecilomyces fumosoroseus Bainier, Paecilomyces sp. Bainier, Verticillium lecani Viegas in $3^{\text {rd }}$ instar larvae and pupae of fruit fly [Ceratitis capitata Wiedermann (Diptera : Tephritidae)]. Initially a selection of those isolates in larvae through the immersion in an aqueous suspension of conidia was made. The larval mortality caused by 36 fungi isolates varied between 2.5 and $96.2 \%$. Six M. anisopliae isolates and two $B$. bassiana isolates were found to be the most pathogenic. In a second series of bioassays, carried out with the selected isolates, pupae were treated in an aqueous suspension at the concentration of $1.2 \times 10^{8}$ viable conidia/mL and germination varied between 85 and $98.2 \%$. Pupae mortality caused by the fungi was low, varying between 1.9 and $4.4 \%$ although the longevity of adults was reduced.
\end{abstract}

Additional keywords: entomopathogenic fungi; fruit flies; microbial control. 


\section{Introdução}

A mosca-das-frutas, Ceratitis capitata Wiedemann (Diptera: Tephritidae), é considerada praga de grande importância econômica para diversas frutíferas no Brasil e no mundo (MALAVASI et al., 2000). O alto potencial reprodutivo e de adaptação, aliado à grande gama de hospedeiros e relativa escassez de inimigos naturais dificultam 0 controle deste inseto (CASTILLO et al., 2000). O método comumente utilizado para seu controle é através do uso de inseticidas químicos, como iscas tóxicas ou em pulverizações em cobertura, podendo causar danos ao meio ambiente e deixar resíduos tóxicos nos frutos.

Uma importante alternativa ao controle químico é o controle microbiano, que tem sido utilizado contra diversas pragas. Alguns fungos deuteromicetos têm sido relatados como patogênicos para dípteros; dentre eles, citam-se Metarhizium anisopliae (Metsch.) Sorokin, Beauveria bassiana (Bals.) Vuillemin, Verticillium lecanii (Zimm.) Viegas e Paecilomyces fumosoroseus Bainier, como os mais importantes (ALVES, 1998a), sendo que, para a mosca-dasfrutas, os fungos mais estudados têm sido $M$. anisopliae e $B$. bassiana. A patogenicidade destes isolados tem sido estudada por vários autores, em diferentes fases do ciclo de vida de $C$. capitata, como exemplo, OLIVEIRA et al. (2010).

Para que se tenha à disposição agentes de alta virulência, para aplicar no controle microbiano de insetos, é importante selecionar organismos que apresentem essa característica, em virtude da variabilidade genética que ocorre dentro de cada espécie.

Este trabalho teve como objetivo verificar a patogenicidade de diferentes espécies de fungos para C. capitata e a seleção dos mais eficientes para larvas e pupas.

\section{Material e métodos}

Ovos de C. capitata foram cedidos pelo Laboratório de Biologia e Criação de Insetos, pertencente à Faculdade de Ciências Agrárias e Veterinárias/UNESP, Câmpus de Jaboticabal-SP, para dar início à criação-estoque. Os insetos foram criados nas dependências do Laboratório de Ecologia Aplicada, Departamento de Fitossanidade, pertencente à mesma Instituição.

A dieta artificial utilizada para criar as larvas foi composta por açúcar, ágar, levedo de cerveja, Nipagin, ácido cítrico e água destilada (DÓRIA, 2001). Os adultos foram confinados em gaiola com armação de metal $(50 \times 50 \times 50 \mathrm{~cm})$, revestida por tela sombrite $(70 \%)$ de insolação e alimentados com uma solução de água destilada e mel a $20 \%$. Os insetos foram mantidos em sala semiclimatizada, com temperatura de $26 \pm 2{ }^{\circ} \mathrm{C}$, umidade relativa do ar de $70 \pm 15 \%$ e fotofase de 12 horas.

As informações a respeito das amostras de fungos constam na Tabela 1. As amostras foram estocadas em freezer, sob temperatura de $-10^{\circ} \mathrm{C}$. Posteriormente, foram multiplicados mediante inoculação em meio BDAL (batata, dextrose, ágar e extrato de levedura) e incubados em câmara climatizada do tipo B.O.D. a $26 \pm 0,5^{\circ} \mathrm{C}$ e 12 horas de fotofase, por 10 dias.

Dois dias antes da aplicação de cada isolado, avaliou-se a viabilidade dos conídios; suspensões aquosas foram distribuídas sobre lâminas de microscopia contendo agar-água (1\%) e incubadas a $26 \pm 0,5{ }^{\circ} \mathrm{C}$. Os valores de germinação foram obtidos a partir de 16 horas de incubação, em microscópio ótico (aumento de 400x). Os resultados foram usados para ajustar as concentrações em conídios viáveis $/ \mathrm{mL}$, sendo os isolados de uma mesma espécie testados na mesma concentração (Tabela 1).

Realizaram-se cinco bioensaios, no total, num período de aproximadamente seis meses: quatro bioensaios para selecionar os isolados mais patogênicos dentre as espécies Metarhizium anisopliae, Beauveria bassiana, Paecilomyces fumosoroseus e Verticillium lecanii, sobre larvas de $3^{0}$ instar, e o último bioensaio para testar os isolados selecionados sobre pupas. Em cada bioensaio, havia uma testemunha, cujos insetos foram imersos em solução de Tween $80^{\circledR}$ a $0,05 \%$.

Em cada tratamento, aplicou-se um isolado, distribuído em quatro repetições, sendo que se utilizaram 20 insetos em cada repetição. Estes indivíduos foram imersos por 10 segundos em suspensão aquosa com concentração entre $1,3 \times 10^{8}$ e $3,1 \times 10^{8}$ conídios viáveis $/ \mathrm{mL}$, conforme metodologia descrita por GUTIÉRREZ et al. (2000). Após a aplicação, os insetos foram confinados em grupo de 20 indivíduos em caixas de plástico de $5 \mathrm{~cm}$ de diâmetro e $2,5 \mathrm{~cm}$ de altura e forradas com papel-filtro estéril umedecido, que foi retirado após 24 horas. As larvas foram mantidas em câmara climatizada do tipo B.O.D, a $26 \pm 0,5^{\circ} \mathrm{C}$ e fotofase de 12 horas.

Realizaram-se avaliações ao completar o $2^{\circ}, 4^{\circ}$ e $6^{\circ}$ dia após a aplicação do fungo. Os insetos mortos foram lavados com álcool a $70 \%$ por 10 segundos, e em seguida em água destilada (MOINO JR. \& ALVES, 1997). Em seguida, foram acondicionados em caixas plásticas vedadas e com algodão umedecido para manter a umidade acima de $90 \%$, para a confirmação da mortalidade pelo fungo. O parâmetro avaliado foi mortalidade confirmada na fase larval e pupal (esporulação do fungo). 
Tabela 1 - Germinação e concentração de conídios de isolados de fungos de diversas procedências, testados em larvas de Ceratitis capitata. Germination and concentration of conidia of fungi isolates from several provenances in larvae of Ceratitis capitata.

\begin{tabular}{|c|c|c|c|c|}
\hline Espécie & Isolado & $\begin{array}{l}\text { Hospedeiro ou } \\
\text { substrato }\end{array}$ & $\begin{array}{c}\text { Germinação } \\
(\%)\end{array}$ & $\begin{array}{c}\text { Concentração } \\
\text { (x } 10^{8} \text { conídios } \\
\text { viáveis } / \mathrm{mL} \text { ) }\end{array}$ \\
\hline Metarhizium anisopliae & IBCB159 ${ }^{(1)}$ & Amostra de solo & 99,3 & 1,3 \\
\hline Metarhizium anisopliae & IBCB $176^{(1)}$ & Amostra de solo & 87,6 & 1,3 \\
\hline Metarhizium anisopliae & IBCB $363^{(1)}$ & Amostra de solo & 80,9 & 1,3 \\
\hline Metarhizium anisopliae & IBCB $368^{(1)}$ & Amostra de solo & 85,0 & 1,3 \\
\hline Metarhizium anisopliae & IBCB $470^{(1)}$ & - & 84,2 & 1,3 \\
\hline Metarhizium anisopliae & IBCB $497^{(1)}$ & - & 81,9 & 1,3 \\
\hline Metarhizium anisopliae & IBCB $535^{(1)}$ & $\begin{array}{l}\text { Larva pão-de- } \\
\text {-galinha }\end{array}$ & 86,5 & 1,3 \\
\hline Metarhizium anisopliae & $\mathrm{E} 9^{(4)}$ & Deois flavopicta & 98,3 & 1,3 \\
\hline Metarhizium anisopliae & IBCamp ${ }^{(1)}$ & - & 99,2 & 1,3 \\
\hline Metarhizium anisopliae & $157 P^{(1)}$ & - & 94,4 & 1,3 \\
\hline Beauveria bassiana & Iplnb1 ${ }^{(3)}$ & - & 93,0 & 2,2 \\
\hline Beauveria bassiana & Inbioter $1^{(3)}$ & - & 94,0 & 2,2 \\
\hline Beauveria bassiana & Inbioter2 ${ }^{(3)}$ & - & 88,0 & 2,2 \\
\hline Beauveria bassiana & JAB06 ${ }^{(4)}$ & - & 93,0 & 2,2 \\
\hline Beauveria bassiana & $\mathrm{JAB} 07^{(4)}$ & - & 98,0 & 2,2 \\
\hline Beauveria bassiana & Am09 ${ }^{(4)}$ & Deois incompleta & 95,0 & 2,2 \\
\hline Beauveria bassiana & IBCB $07^{(1)}$ & - & 96,2 & 2,2 \\
\hline Beauveria bassiana & IBCB66 ${ }^{(1)}$ & - & 90,1 & 2,2 \\
\hline Beauveria bassiana & Esalq $634^{(2)}$ & Solenopsis sp. & 81,3 & 2,2 \\
\hline Beauveria bassiana & Esalq531 ${ }^{(2)}$ & Solenopsis invicta & 83,1 & 2,2 \\
\hline Paecilomyces fumosoroseus & IBCB118 ${ }^{(1)}$ & Amostra de solo & 98,0 & 3,1 \\
\hline Paecilomyces fumosoroseus & IBCB124 ${ }^{(1)}$ & Amostra de solo & 98,7 & 3,1 \\
\hline Paecilomyces fumosoroseus & IBCB127 ${ }^{(1)}$ & Amostra de solo & 96,7 & 3,1 \\
\hline Paecilomyces fumosoroseus & IBCB130 (1) & Amostra de solo & 98,4 & 3,1 \\
\hline Paecilomyces fumosoroseus & IBCB132 ${ }^{(1)}$ & Amostra de solo & 96,6 & 3,1 \\
\hline Paecilomyces fumosoroseus & IBCB133 ${ }^{(1)}$ & Amostra de solo & 97,6 & 3,1 \\
\hline Paecilomyces fumosoroseus & IBCB137 ${ }^{(1)}$ & Amostra de solo & 98,2 & 3,1 \\
\hline Paecilomyces fumosoroseus & IBCB139 (1) & Amostra de solo & 96,6 & 3,1 \\
\hline Paecilomyces fumosoroseus & IBCB148 ${ }^{(1)}$ & Amostra de solo & 98,1 & 3,1 \\
\hline Paecilomyces fumosoroseus & IBCB201 ${ }^{(1)}$ & Amostra de solo & 94,0 & 3,1 \\
\hline Paecilomyces sp. & JAB14 ${ }^{(3)}$ & Bemisia tabaci & 83,7 & 3,1 \\
\hline Verticillium lecanii & $\mathrm{JAB02}{ }^{(3)}$ & Coccus viridis & 96,8 & 2,0 \\
\hline Verticillium lecanii & JAB45 ${ }^{(3)}$ & Coccus viridis & 99,5 & 2,0 \\
\hline Verticillium lecanii & $\mathrm{CAPCH} 1^{(3)}$ & $\begin{array}{l}\text { Leptopharsa } \\
\text { heveae }\end{array}$ & 91,8 & 2,0 \\
\hline Verticillium lecanii & $\mathrm{CAPCH} 2^{(3)}$ & $\begin{array}{l}\text { Leptopharsa } \\
\text { heveae }\end{array}$ & 98,5 & 2,0 \\
\hline Verticillium lecanii & $\mathrm{CAPCH} 3^{(3)}$ & $\begin{array}{l}\text { Leptopharsa } \\
\text { heveae }\end{array}$ & 93,4 & 2,0 \\
\hline
\end{tabular}


Com base nos resultados dos quatro primeiros bioensaios, selecionaram-se os isolados que atingiram mortalidade acima de $90 \%$ (seis de $M$. anisopliae e dois de B. bassiana) para testá-los em pupas. A imersão das pupas nas suspensões foi realizada pelo mesmo método descrito anteriormente.

Após a emergência dos adultos neste último bioensaio, acompanhou-se a mortalidade a cada 5 dias, totalizando 30 dias de avaliação. Os adultos que emergiram foram confinados em potes de plástico de $250 \mathrm{~mL}$, fechados na parte superior com tecido "voile" e foram alimentados com solução de água e mel a $20 \%$ (DÓRIA, 2001), oferecida em pedaços de esponja. As pupas mortas passaram pelo mesmo procedimento de assepsia e confirmação da mortalidade pelo fungo realizado nas larvas.

Para a avaliação da mortalidade de larvas, pupas e adultos, utilizou-se do delineamento experimental inteiramente casualizado, com quatro repetições. Os dados de porcentagem de mortalidade foram transformados em ARCOSENO $\sqrt{(P / 100)}$ com o objetivo de normalizar os dados e homogeneizar as variâncias dos tratamentos. Os dados foram submetidos à análise de variância, pelo teste $F$, e as médias foram comparadas pelo teste de Tukey, a $5 \%$ de probabilidade.

\section{Resultados e discussão}

Os isolados de $M$. anisopliae causaram altos índices de mortalidade total (larval + pupal) em C. capitata (Tabela 2), nas concentrações testadas. O menos patogênico dos isolados, 0 CB368, matou $60,0 \%$ dos insetos.

Os maiores índices de mortalidade larval foram observados quatro dias após a aplicação do patógeno, sendo que, no sexto dia, praticamente não se verificaram novas mortes de larvas. Verificou-se, também, mortalidade pupal. Para confirmar a mortalidade pelo fungo, foi realizada assepsia nas pupas inviáveis e acondicionamento em placas de Petri, conforme descrito anteriormente. Esse fato foi observado aos 15 dias após a aplicação do fungo (DAA).

A alta mortalidade causada por $M$. anisopliae pode ser atribuída, em parte, à liberação de proteínas que atuam no processo de infecção e morte do inseto. ORTIZ-URQUIZA et al. (2009) relatam a descoberta de algumas proteínas extraídas de $M$. anisopliae, que estão envolvidas com a atividade inseticida contra $C$. capitata. NEVES \& ALVES (2004) verificaram que, no desenvolvimento externo de $M$. anisopliae e $B$. bassiana sobre o corpo de Corniternes cumulans, havia vários pontos de penetração que se originaram de um único tubo germinativo, e que este fator pode estar associado à maior virulência do fungo.

Tabela 2 - Porcentagem de mortalidade confirmada ${ }^{(1)}$ de Ceratitis capitata após imersão das larvas em suspensão aquosa de conídios de Metarhizium anisopliae. Confirmed mortality (\%) of Ceratitis capitata after the larvae were immersed in an aqueous suspension of Metarhizium anisopliae conidia.

\begin{tabular}{|c|c|c|c|}
\hline \multirow{2}{*}{ Isolado } & \multicolumn{3}{|c|}{ Dias após a aplicação } \\
\hline & 4 & 6 & 15 \\
\hline $157 P$ & $76,2 \pm 8,50 a$ & $76,2 \pm 8,50 a$ & $96,2 \pm 3,75 a$ \\
\hline E9 & $66,2 \pm 3,75 a$ & $66,2 \pm 3,75 a b$ & $95,0 \pm 5,00 a$ \\
\hline IBCB470 & $48,7 \pm 9,86 a$ & $48,7 \pm 9,86 a b$ & $96,2 \pm 2,39 a$ \\
\hline IBCB497 & $75,0 \pm 7,90 a$ & $75,0 \pm 7,90 a b$ & $96,2 \pm 1,25 a$ \\
\hline IBCB363 & $52,5 \pm 2,50 a$ & $52,5 \pm 2,50 a b$ & $93,7 \pm 3,75 a$ \\
\hline IBCB535 & $43,7 \pm 10,15 a$ & $43,7 \pm 10,07 a b$ & $91,2 \pm 3,14 a b$ \\
\hline IBCB159 & $51,2 \pm 18,52 a$ & $57,5 \pm 13,76 a b$ & $86,2 \pm 8,00 a b$ \\
\hline IBCamp & $47,5 \pm 4,78 \mathrm{a}$ & $47,5 \pm 4,78 a b$ & $83,7 \pm 3,14 a b$ \\
\hline IBCB176 & $36,2 \pm 6,57 \mathrm{a}$ & $36,2 \pm 6,57 b$ & $72,5 \pm 5,95 a b$ \\
\hline IBCB368 & $36,2 \pm 8,26 a$ & $36,2 \pm 8,26 b$ & $60,0 \pm 6,45 b$ \\
\hline Teste F & 2,68 * & $3,15^{* *}$ & $4,24^{* *}$ \\
\hline DMS (Tukey, 5\%) & 32,03 & 29,71 & 35,74 \\
\hline $\begin{array}{l}\text { (1) Mortalidade confirmada } \\
\text { sinais do patógeno; índic } \\
\text { fungos. (2) Médias na } \\
\text { ARCOSENO } \sqrt{(P / 100)} \\
\text { pelo teste de Tukey }(P>0\end{array}$ & $\begin{array}{l}\text { que após sofrer } \\
\text { talidade na teste } \\
\text { ginal e análise } \\
\text { EP) seguidas pc }\end{array}$ & $\begin{array}{l}\text { psia e mantidos e } \\
\text { le } 11,2 \% \text { e não foi } \\
\text { ncia realizada cor } \\
\text { menos, uma letra } €\end{array}$ & $\begin{array}{l}\text { a úmida, apreser } \\
\text { do contaminação } \\
\text { dos transformad } \\
\text { m não diferem el } \\
\text { mente. }\end{array}$ \\
\hline
\end{tabular}


Tabela 3 - Porcentagem de mortalidade confirmada $^{(1)}$ de Ceratitis capitata após imersão das larvas em suspensão aquosa de conídios de Beauveria bassiana. Confirmed mortality (\%) of Ceratitis capitata after the larvae were immersed in an aqueous suspension of Beauveria bassiana conidia.

\begin{tabular}{lccc}
\hline \multirow{2}{*}{ Isolado } & \multicolumn{3}{c}{ Dias após a aplicação } \\
\cline { 2 - 4 } & 4 & 6 & 15 \\
\hline Esalq 634 & $10,0 \pm 3,22 \mathrm{ab}$ & $33,7 \pm 13,28 \mathrm{a}$ & $92,5 \pm 3,22 \mathrm{a}$ \\
Esalq 531 & $12,5 \pm 0,00 \mathrm{a}$ & $23,7 \pm 6,88 \mathrm{ab}$ & $80,0 \pm 4,56 \mathrm{a}$ \\
IBCB 07 & $3,7 \pm 2,39 \mathrm{ab}$ & $12,5 \pm 7,5 \mathrm{ab}$ & $38,7 \pm 7,8 \mathrm{~b}$ \\
Inbioter2 & $0,0 \pm 0,00 \mathrm{~b}$ & $8,7 \pm 7,18 \mathrm{ab}$ & $32,5 \pm 8,29 \mathrm{~b}$ \\
Iplnb1 & $0,0 \pm 0,00 \mathrm{~b}$ & $7,5 \pm 3,22 \mathrm{ab}$ & $25,0 \pm 4,56 \mathrm{~b}$ \\
Inbioter1 & $0,0 \pm 0,00 \mathrm{~b}$ & $16,2 \pm 10,05 \mathrm{ab}$ & $23,7 \pm 14,19 \mathrm{~b}$ \\
Jab06 & $1,2 \pm 1,25 \mathrm{ab}$ & $6,2 \pm 3,14 \mathrm{ab}$ & $21,2 \pm 4,73 \mathrm{~b}$ \\
Am09 & $1,2 \pm 1,25 \mathrm{ab}$ & $6,2 \pm 1,25 \mathrm{ab}$ & $17,5 \pm 4,33 \mathrm{~b}$ \\
Jab07 & $0,0 \pm 0,00 \mathrm{~b}$ & $3,7 \pm 1,25 \mathrm{~b}$ & $12,5 \pm 3,22 \mathrm{~b}$ \\
IBCB66 & $0,0 \pm 0,00 \mathrm{~b}$ & $0,0 \pm 0,00 \mathrm{~b}$ & $7,5 \pm 1,44 \mathrm{~b}$ \\
\hline Teste F & $3,22{ }^{* *}$ & $2,45{ }^{*}$ & 21,55 ** \\
DMS (Tukey, 5\%) & 7,14 & 19,64 & 22,71 \\
\hline
\end{tabular}

(1) Mortalidade confirmada: insetos que, após sofrerem assepsia e mantidos em câmara úmida, apresentaram sinais do patógeno; índice de mortalidade na testemunha de 5,0\% e não foi observada contaminação pelos fungos. (2) Médias na escala original e análise de variância realizada com os dados transformados em ARCOSENO $\sqrt{(P / 100)}$; médias $( \pm \mathrm{EP})$ seguidas por, pelo menos, uma letra em comum não diferem entre si, pelo teste de Tukey $(\mathrm{P}>0,05) ;{ }^{*} \mathrm{e}^{* *}$ Significativo a $5 \%$ e $1 \%$ de probabilidade, respectivamente.

Em alguns estudos realizados por GUTIÉRREZ et al. (2000) e OLIVEIRA et al. (2010), verificou-se alta virulência de $M$. anisopliae para larvas e pupas de espécies de mosca-das-frutas. No atual trabalho, $M$. anisopliae foi a espécie que causou maior índice de mortalidade. DESTÉFANO et al. (2005) também encontraram altos índices de mortalidade de Anastrepha fraterculus quando tratadas com o isolado E9 (M. anisopliae).

No bioensaio com $B$. bassiana também ocorreram diferenças nos índices de mortalidade entre isolados (Tabela 3). A mortalidade até o $2^{\circ}$ DAA foi praticamente nula, semelhante ao verificado com $M$. anisopliae. No $4^{\circ}$ DAA, a mortalidade aumentou, mas mesmo assim não ultrapassou $12,5 \%$. Os maiores índices foram constatados no sexto dia $(33,75 \%)$. Considerando as mortalidades totais acumuladas (larva + pupas), Esalq634 e Esalq531 foram os isolados mais patogênicos com 92,5 e 80,0\% de mortalidade, respectivamente. Os outros causaram mortalidades baixas, que não chegaram a atingir $40,0 \%$.

DANIEL \& WYSS (2009) verificaram que, dentre diversas espécies de patógenos, $B$. bassiana foi a que mais se destacou, quando testados em mosca-das-frutas da espécie Rhagoletis cerasi. COSSENTINE et al. (2010) também verificaram que $B$. bassiana causou alto índice de mortalidade larval em Rhagoletis indifferens.

QUESADA-MORAGA et al. (2006), ao testarem diferentes isolados de $B$. bassiana e $M$. anisopliae sobre pupas e adultos de C. capitata, verificaram que todos os isolados testados mostraram-se patogênicos, demonstrando o potencial promissor destas espécies de fungos para o controle biológico de mosca-das-frutas.

Para P. fumosoroseus (Tabela 4) e $V$. lecanii (Tabela 5), as porcentagens de mortalidade foram baixas nas concentrações testadas, e não apresentaram diferenças significativas entre os isolados testados. Os maiores índices de mortalidade atingidos para $P$. fumosoroseus foi de $12,5 \%$ (IBCB118), e para V. lecanii, $17,5 \%$ (CPACH1).

CASTILLO et al. (2000) e ALI et al. (2008) observaram que, entre outras espécies, $P$. fumosoroseus provocou altos índices de mortalidade quando aplicado em adultos de $C$. capitata em condições de laboratório. Isso demonstra que pode haver diferença na suscetibilidade do inseto ao entomopatógeno, de acordo com o estádio biológico em que sofre a infecção. Por outro lado, ANAGNOU-VERONIKI (2005) verificou baixo índice de mortalidade $(11,3 \%)$ de adultos de Bactrocera oleae, após aplicação de produto comercial à base de $V$. lecanii.

Os isolados selecionados (IBCB497, E9, 157P, IBCB535, IBCB363, CB634, CB531) foram aplicados em pupas, na concentração de 1,2 $\mathrm{x}$ $10^{8}$ conídios viáveis $/ \mathrm{mL}$ e germinação variando de 85 a 98,2\%. Verificou-se que os índices de mortalidade pupal foram baixos, variando de 4,4 a $11,3 \%$ (Teste $F=0,57$ - não significativo), não ocorrendo diferença significativa em relação à 
testemunha. Talvez isto se deva a defesas mecânicas da pupa, aliadas a mecanismos celula-

res e/ou bioquímicos contra entomopatógenos (OMOTO \& ALVES, 1998).

Tabela 4 - Porcentagem de mortalidade confirmada ${ }^{1}$ de Ceratitis capitata após imersão das larvas em suspensão aquosa de conídios de Paecilomyces fumosoroseus. Confirmed mortality (\%) of Ceratitis capitata after the larvae were immersed in aqueous suspension of Paecilomyces fumosoroseus conidia.

\begin{tabular}{lccr}
\hline \multirow{2}{*}{ Isolado } & \multicolumn{3}{c}{ Dias após a aplicação } \\
\cline { 2 - 4 } & 4 & 6 & 15 \\
\hline IBCB118 & $2,5 \pm 1,44 \mathrm{a}$ & $2,8 \pm 1,44 \mathrm{a}$ & $12,5 \pm 4,78 \mathrm{a}$ \\
IBCB124 & $0,0 \pm 0,00 \mathrm{a}$ & $0,0 \pm 0,00 \mathrm{a}$ & $5,0 \pm 2,04 \mathrm{a}$ \\
IBCB127 & $0,0 \pm 0,00 \mathrm{a}$ & $0,0 \pm 0,00 \mathrm{a}$ & $6,2 \pm 4,73 \mathrm{a}$ \\
IBCB130 & $5,0 \pm 2,04 \mathrm{a}$ & $5,0 \pm 2,04 \mathrm{a}$ & $5,0 \pm 2,04 \mathrm{a}$ \\
IBCB132 & $0,0 \pm 0,00 \mathrm{a}$ & $0,0 \pm 0,00 \mathrm{a}$ & $5,0 \pm 3,53 \mathrm{a}$ \\
IBCB133 & $1,2 \pm 1,25 \mathrm{a}$ & $1,2 \pm 1,25 \mathrm{a}$ & $10,0 \pm 2,04 \mathrm{a}$ \\
IBCB137 & $1,2 \pm 1,25 \mathrm{a}$ & $1,2 \pm 1,25 \mathrm{a}$ & $11,2 \pm 2,35 \mathrm{a}$ \\
IBCB139 & $3,7 \pm 1,25 \mathrm{a}$ & $5,0 \pm 2,04 \mathrm{a}$ & $11,2 \pm 5,15 \mathrm{a}$ \\
IBCB148 & $2,5 \pm 2,5 \mathrm{a}$ & $2,5 \pm 2,50 \mathrm{a}$ & $3,7 \pm 2,39 \mathrm{a}$ \\
IBCB201 & $1,2 \pm 1,25 \mathrm{a}$ & $1,2 \pm 1,25 \mathrm{a}$ & $5,0 \pm 2,88 \mathrm{a}$ \\
\hline Teste F & $1,522^{\mathrm{NS}}$ & $1,67^{\mathrm{NS}}$ & $0,95^{\mathrm{NS}}$ \\
DMS (\%) & 3,79 & 4,04 & 9,51
\end{tabular}

(1) Mortalidade confirmada: insetos que, após sofrerem assepsia e mantidos em câmara úmida, apresentaram sinais do patógeno; índice de mortalidade na testemunha de 10,0\% e não foi observado contaminação pelos fungos. (2) Médias na escala original e análise de variância realizada com os dados transformados em ARCOSENO $\sqrt{(P / 100)}$; médias ( $\pm E P)$ seguidas por, pelo menos, uma letra em comum não diferem entre si, pelo teste de Tukey $(P>0,05)$; NS Não significativo.

Tabela 5 - Porcentagem de mortalidade confirmada ${ }^{1}$ de Ceratitis capitata após imersão das larvas em suspensão aquosa de conídios de Verticillium lecanii. Confirmed ${ }^{1}$ mortality (\%) of Ceratitis capitata after the larvae were immersed in aqueous suspension of Verticillium lecani conidia.

\begin{tabular}{lccc}
\hline Isolado & \multicolumn{3}{c}{ Dias após a aplicação } \\
\cline { 2 - 4 } & 4 & 6 & 15 \\
\hline CPACH1 & $3,75 \pm 3,7 \mathrm{a}$ & $3,75 \pm 3,7 \mathrm{a}$ & $17,50 \pm 11,0 \mathrm{a}$ \\
CAPCH2 & $1,25 \pm 1,2 \mathrm{a}$ & $1,25 \pm 1,2 \mathrm{a}$ & $7,50 \pm 3,2 \mathrm{a}$ \\
CPACH3 & $0,00 \pm 0,0 \mathrm{a}$ & $0,00 \pm 0,0 \mathrm{a}$ & $2,50 \pm 1,4 \mathrm{a}$ \\
Jab02 & $0,00 \pm 0,0 \mathrm{a}$ & $2,50 \pm 1,4 \mathrm{a}$ & $5,00 \pm 2,0 \mathrm{a}$ \\
Jab45 & $0,00 \pm 0,0 \mathrm{a}$ & $0,00 \pm 0,0 \mathrm{a}$ & $13,75 \pm 4,7 \mathrm{a}$ \\
\hline Teste F & $0,85^{\text {NS }}$ & $0,75^{\text {NS }}$ & $1,18^{\mathrm{NS}}$ \\
DMS (\%) & $4,44^{2}$ & 4,72 & 14,82
\end{tabular}

(1) Mortalidade confirmada: insetos que, após sofrerem assepsia e mantidos em câmara úmida, apresentaram sinais do patógeno; índice de mortalidade na testemunha de 13,7\% e não foi observado contaminação pelos fungos. (2) Médias na escala original e análise de variância realizada com os dados transformados em ARCOSENO $\sqrt{(P / 100)}$; médias ( \pm EP) seguidas por, pelo menos, uma letra em comum não diferem entre si, pelo teste de Tukey $(P>0,05)$. NS Não significativo.

Embora os isolados selecionados não tenham afetado significativamente a viabilidade das pupas, observou-se menor longevidade dos adultos provenientes das pupas tratadas, comparada com a testemunha (Tabela 6 ). Isso mostra que, mesmo o fungo sendo aplicado na fase de pupa, pode afetar a longevidade dos adultos. Os isolados que se destacaram neste aspecto foram: CB497, CB470, E9 e 157P, todos de $M$. anisopliae.

Resultado semelhante foi encontrado por QUESADA-MORAGA et al. (2006) e EVANGELOS et al. (2013). Ao testarem diferen-

tes isolados de fungos entomopatogênicos sobre pupas de $C$. capitata, obtiveram baixos índices de mortalidade pupal, porém os adultos provenientes das pupas tratadas tiveram altos índices de mortalidade comparada com a testemunha. DIMBI et al. (2003) também verificaram que a idade do inseto afeta diretamente a suscetibilidade ao entomopatógeno $M$. anisopliae.

ALVES (1998b) relatou que a patogenicidade não deve ser verificada somente pela mortalidade na população, mas também por diversos efeitos na fisiologia ou biologia dos insetos, entre eles a longevidade. Segundo o 
autor, a ação de toxinas produzidas por microrganismos pode gerar um estado doentio, com sintomas difíceis de serem caracterizados.

Verificou-se também a emergência dos fungos em insetos adultos, após terem sofrido assepsia e serem condicionados em câmara úmida, comprovando que a morte foi causada pelo patógeno.
Os resultados obtidos podem ser utilizados como estratégia de controle biológico para a redução populacional de $C$. capitata no ambiente agrícola. Para isso, há necessidade de testes de campo para avaliar a eficiência destes isolados, aplicando-os ao solo em condições naturais.

Tabela 6 - Porcentagem de mortalidade de adultos de Ceratitis capitata, oriundos de pupas tratadas com fungos entomopatogênicos. Ceratitis capitata adults mortality originated from pupae treated with entomopathogenic fungi.

\begin{tabular}{llllllll}
\multirow{2}{*}{ Isolado } & \multicolumn{7}{c}{ Dias após a emergência de adultos } \\
\cline { 2 - 8 } & \multicolumn{1}{c}{1} & 5 & 10 & 15 & 20 & 25 & 30 \\
\hline Ma IBCB497 & $3,2^{1} \mathrm{ab}^{2}$ & $21,0 \mathrm{a}$ & $78,1 \mathrm{a}$ & $92,5 \mathrm{a}$ & $93,8 \mathrm{ab}$ & $97,6 \mathrm{a}$ & $99,3 \mathrm{a}$ \\
Ma IBCB470 & $3,2 \mathrm{ab}$ & $14,3 \mathrm{ab}$ & $83,8 \mathrm{a}$ & $92,5 \mathrm{a}$ & $94,1 \mathrm{a}$ & $96,7 \mathrm{a}$ & $99,3 \mathrm{a}$ \\
Ma E9 & $0,0 \mathrm{~b}$ & $17,6 \mathrm{a}$ & $85,0 \mathrm{a}$ & $90,0 \mathrm{a}$ & $92,5 \mathrm{ab}$ & $93,2 \mathrm{a}$ & $93,8 \mathrm{a}$ \\
Ma 157P & $6,0 \mathrm{a}$ & $22,0 \mathrm{a}$ & $73,1 \mathrm{a}$ & $85,0 \mathrm{a}$ & $88,9 \mathrm{abc}$ & $97,1 \mathrm{a}$ & $99,3 \mathrm{a}$ \\
Ma IBCB535 & $0,0 \mathrm{~b}$ & $10,7 \mathrm{abc}$ & $19,0 \mathrm{bc}$ & $46,4 \mathrm{~b}$ & $71,7 \mathrm{bc}$ & $81,7 \mathrm{a}$ & $89,3 \mathrm{a}$ \\
Ma IBCB363 & $0,0 \mathrm{~b}$ & $5,6 \mathrm{bc}$ & $16,5 \mathrm{bc}$ & $50,3 \mathrm{~b}$ & $67,3 \mathrm{c}$ & $83,3 \mathrm{a}$ & $93,1 \mathrm{a}$ \\
Bb IBCB634 & $0,6 \mathrm{~b}$ & $16,1 \mathrm{ab}$ & $43,3 \mathrm{~b}$ & $66,4 \mathrm{ab}$ & $79,3 \mathrm{abc}$ & $87,9 \mathrm{a}$ & $94,3 \mathrm{a}$ \\
Bb IBCB531 & $0,0 \mathrm{~b}$ & $15,6 \mathrm{ab}$ & $42,5 \mathrm{~b}$ & $76,6 \mathrm{ab}$ & $86,4 \mathrm{abc}$ & $90,9 \mathrm{a}$ & $96,1 \mathrm{a}$ \\
Testemunha & $0,0 \mathrm{~b}$ & $5,0 \mathrm{c}$ & $16,0 \mathrm{c}$ & $68,5 \mathrm{ab}$ & $73,7 \mathrm{abc}$ & $82,6 \mathrm{a}$ & $93,5 \mathrm{a}$ \\
\hline Teste F & $5,04^{* *}$ & $6,35^{\star *}$ & $24,90^{* *}$ & $8,35^{\star *}$ & $3,93^{\star *}$ & $3,20^{\star *}$ & $1,93^{\mathrm{NS}}$ \\
DMS & 7,63 & 3,49 & 17,78 & 20,08 & 19,70 & 17,66 & 15,43
\end{tabular}

(1) Médias na escala original e análise de variância realizada com os dados transformados em ARCOSENO $\sqrt{(P / 100)}$; médias seguidas por, pelo menos, uma letra em comum não diferem entre si, pelo teste de Tukey $(P>0,05)$. NS Não significativo. ${ }^{*} e^{* *}$ Significativo a $5 \%$ e $1 \%$ de probabilidade, respectivamente. Índice de mortalidade na testemunha de $8,0 \%$ e não foi observado contaminação pelos fungos.

\section{Conclusões}

Os isolados mais patogênicos para larvas de $C$. capitata pertencem às espécies $M$. anisopliae (IBCB497, IBCB470, E9, IBCB535, IBCB363) e B. bassiana (IBCB634 e IBCB531).

Pupas de $C$. capitata são pouco suscetíveis aos isolados selecionados das espécies de $M$. anisopliae e $B$. bassiana, anteriormente citados, nas concentrações testadas. Porém, reduzem significativamente a longevidade de adultos.

\section{Agradecimentos}

Ao Prof. Dr. Odair Aparecido Fernandes, por ceder as instalações de seu laboratório para a criação- estoque dos insetos e pelas sugestões feitas no manuscrito; ao Prof. Dr. Antônio Carlos Monteiro, pelas críticas feitas ao manuscrito e à pós-graduanda Hayda O. S. Dória, por ter doado os ovos de C. capitata para início da criação; ao CNPq, pela concessão de bolsa de estudos ao primeiro autor, durante o curso de Mestrado na área de Entomologia Agrícola na FCAV/UNESP, Câmpus de Jaboticabal, que tornou possível a realização desta pesquisa.

\section{Referências}

ALI, A.; SERMANN, H.; BÜTTNER, C. Susceptibility of Ceratitis capitata Wiedemann (Diptera: Tephritidae) to entomopathogenic fungi. Communications in agricultural and applied biological sciences, Bélgica, v.73, n.3, p.589-596, 2008.

ALVES, S. B. Fungos entomopatogênicos. In: ALVES, S. B. Controle microbiano de insetos. 2.ed. Piracicaba: FEALQ, 1998a. p.289-380.

ALVES, S. B. Microorganismos associados a insetos. In: ALVES, S. B. Controle microbiano de insetos. 2. ed. Piracicaba: FEALQ, 1998b. p.75-94.

ANAGNOU-VERONIKI, M.; KONTODIMAS, D. C.; ADAMOPOULOS, A. D.; TSIMBOUKIS, N. D.; AND A. VOULGAROPOULOU. Integrated Protection of Olive Crops, Grécia, v.28, n.9, p.49-51, 2005.

CASTILLO, M. A.; MOYA, P.; HERNÁNDEZ, E.; YÚFERA, E. P. Susceptibility of Ceratitis capitata Wiedemann (Diptera: Tephritidae) to entomopathogenic fungi and their extracts. 
Biological Control, San Diego, v.19, n.3, p.274282, 2000.

COSSENTINE, J.; THISTLEWOOD, H.; GOETTEL, M.; JARONSKI, S. Susceptibility of preimaginal western cherry fruit fly, Rhagoletis indifferens (Diptera: Tephritidae) to Beauveria bassiana (Balsamo) Vuillemin Clavicipitaceae (Hypocreales). Journal of Invertebrate Pathology, New York, v.104, n.2, p.105-109, 2010.

DANIEL, C.; WYSS, E. Susceptibility of different life stages of the European cherry fruit fly, Rhagoletis cerasi, to entomopathogenic fungi. Journal of Applied Entomology, Berlin, v.133, n.6, p.473-483, 2009.

DESTÉFANO, R. H. R; BECHARA, I. J.; MESSIAS, C. L.; PIEDRABUENA, A. E. Effectiveness of Metarhizium anisopliae against immature stages of Anastrepha fraterculus fruit fly (Diptera: Tephritidae). Brazilian Journal of Microbiology, São Paulo, v.36, n.1, p.94-99, 2005.

DIMBI, S.; MANIANIA, N. K.; LUX, S. A.; MUEKE, J. M. Host species, age and sex as factors affecting the susceptibility of the African Tephritid fruit fly species, Ceratitis capitata, $\boldsymbol{C}$. cosyra and $C$. fasciventris to infection by Metarhizium anisopliae. Journal of Pest Science, Verlag, v.76, n.5, p.113-117, 2003.

DÓRIA, H. O. S. Influência do tratamento térmico com vapor e água quente na mortalidade de mosca-das-frutas e qualidade de frutos de goiaba (Psidium guajava L.). 2001. 73f. Dissertação (Mestrado em Entomologia Agrícola) - Faculdade de Ciências Agrárias e Veterinárias, Universidade Estadual Paulista, Jaboticabal, 2001.

EVANGELOS, I.B.; DIMITRIOS, P. P.; ANASTASIA, F.; SPYRIDON, A. A.; DIMITRIOS, C. K.; Pathogenicity of three entomopathogenic fungi on pupae and adults of the Mediterranean fruit fly, Ceratitis capitata (Diptera: Tephritidae). Journal of Pest Science, Berlin, v.86, n.2, p.275-284, 2013.

GUTIÉRREZ, R. L.; DE-LA-LUZ, A. T.; OCHOA, J. M.; DOMINGUEZ, O. R.; PESCADOR, A. R.; EDWARDS, M. L.; ALUJA, M. Virulence of Metarhizium anisoplie (Deuteromycotina: Hyphomycetes) on Anastrepha ludens (Diptera: Tephritidae): Laboratory and field trials. Biological and Microbial Control, Lanham, v.93, n.4, p.1080-1084, 2000.
MALAVASI, A.; ZUCCHI, R. A.; SUGAYAMA, R. L. Biogeografia. In: MALAVASI, A.; ZUCCHI, R. A. Moscas-das-frutas de importância econômica no Brasil: conhecimento básico e aplicado. Ribeirão Preto: Holos Editora, 2000, p.93-98.

MOINO JÚNIOR, A.; ALVES, S. B. Determinação de concentrações de Beauveria bassiana (Bals.) Vuill. para o controle de insetos-praga de grãos armazenados. Anais da Sociedade Entomológica do Brasil, Itabuna, v.26, n.1, p.15-20, 1997.

NEVES, P. M. O. J.; ALVES. S. B. Eventos externos relacionados ao processo de infecção de Cornitermes cumulans (Kollar) (Isoptera:Termitidae) pelos fungos entomopatogênicos Beauveria bassiana e Metarhizium anisopliae. Neotropical Entomology, Londrina, v.33, n.1, p.51-56, 2004.

OLIVEIRA ${ }^{1}$, F. Q.; BATISTA, J. L.; MALAQUIAS, J. B.; ALMEIDA, D. M.; OLIVEIRA, R. Determination of the median lethal concentration $\left(\mathrm{LC}_{50}\right)$ of mycoinsecticides for the control of Ceratitis capitata (Diptera: Tephritidae). Revista Colombiana de Entomologia, Bogotá, v.36, n.2, p.213-216, 2010.

OMOTO C.; ALVES, S. B. Mecanismos de defesa de insetos contra entomopatógenos. In: ALVES, S. B. Controle microbiano de insetos. 2. ed. Piracicaba: FEALQ, 1998. p.55-70.

ORTIZ-URQUIZA A.; GARRIDO-JURADO, I.; SANTIAGO-ÁLVAREZ, C.; QUESADA-MORAGA, E. Purification and characterisation of proteins secreted by the entomopathogenic fungus Metarhizium anisopliae with insecticidal activity against adults of the Mediterranean fruit fly, Ceratitis capitata (Diptera: Tephritidae). Pest Management Science, New York, v.65, n.10, p.1130-1139, 2009.

QUESADA-MORAGA, E.; RUIZ-GARCÍA, A.; SANTIAGO-ÁLVAREZ, C. Laboratory evaluation of entomopathogenic fungi Beauveria bassiana and Metarhizium anisopliae against puparia and adults of Ceratitis capitata (Diptera: Tephritidae). Journal of Economic Entomology, Lanham, v.99, n.6, p.1955-1966, 2006. 